\title{
BRIBRI, NUEVA VARIEDAD DE FRIJOL ROJO PEQUEÑO PARA COSTA RICA ${ }^{1}$
}

\author{
Juan Carlos Hernández ${ }^{2}$, Rodolfo Araya ${ }^{3}$, Adrián Morales 4
}

\section{RESUMEN}

Bribri, nueva variedad de frijol rojo pequeño para Costa Rica. Entre 1996 y 1999 fue introducida y evaluada en Costa Rica la línea MD 23-24 que dio origen a la nueva variedad de frijol Bribri. La misma fue desarrollada por la Escuela Agrícola Panamericana-Zamorano y se originó de la cruza (RAB 310/XAN 155) X (DOR 391/POMPADOUR G). La variedad se caracteriza por presentar un grano pequeño de color rojo brillante, de hábito de crecimiento Tipo II-B, de porte erecto y ciclo vegetativo entre 76 y 80 días. Las dos principales zonas de vida donde se evaluó fueron la bh-T (bosque húmedo tropical) y el bmh-P (bosque muy húmedo Premontano). En la mayoría de los lugares evaluados predominaron pequeñas explotaciones comerciales y suelos ultisoles de baja fertilidad. La variedad Bribri superó en rendimiento promedio, al testigo nacional en $17,2 \%$ y al testigo local en $13,4 \%$. Su producción fue superior en un $8,5 \%$ al promedio de rendimiento obtenido en todos los ensayos. La etapa de verificación y de validación se efectuó en nueve y once localidades respectivamente, con base en la tecnología empleada por los productores. Bribri superó en rendimiento a los testigos locales en el $67 \%$ de las parcelas en la fase de verificación (con un promedio de $15,5 \%$ más de $\mathrm{kg} / \mathrm{ha}$ ) y en el $80 \%$ de las parcelas de validación (con un promedio de 7,5\% mas de $\mathrm{kg} / \mathrm{ha}$ ). Mostró además tolerancia a la mustia hilachosa. (Thanatephorus cucumeris (A.B. Frank) Donk). En el año 2000 se sembraron 100 ha de Bribri a nivel comercial en la Región Brunca.

\begin{abstract}
Bribri, a new small red bean variety for Costa Rica. The line MD 23-24 was introduced and tested in Costa Rica between 1996 and 1999, which served as the precursor of the Bribri bean variety. It was bred, at the Escuela Agrícola Panamericana Zamorano, by crossing (RAB 310/XAN 155) X (DOR 391/POMPADOUR G). The variety characterizes by showing a small bright red grain, with a II-B Type growth habit, an erect bearing and a vegetative cycle from 76-80 days. The two main life zones where it was evaluated were the bh-T (tropical humid forest) and the bmh-P (very humid pre-mountainous forest). Small commercial farms with low fertility soils prevailed in most of the evaluated localities. The Bribri variety outstood in average yield by $17.2 \%$ the national control and by $13,4 \%$ the local control. Its yield was $8.5 \%$ higher than the average yield of all the assays. The verifying and the validation stages were conducted based on the technology applied by the farmers in nine out of eleven localities. Bribri out-yielded the local controls in $67 \%$ of the plots at the verifying phase (with an average of $15.5 \%$ more $\mathrm{kg} / \mathrm{ha}$ ) and in $80 \%$ of the validation plots (with an average of $7.5 \%$ more $\mathrm{kg} / \mathrm{ha}$ ). Besides, it showed tolerance to web-blight (Thanatephorus cucumeris (A.B. Frank) Donk).
\end{abstract}

\section{INTRODUCCION}

El Programa de Investigación y Transferencia de Tecnología Agropecuaria en Frijol (PITTA-Frijol), ha mantenido durante 24 años un programa cooperativo entre las diferentes instituciones involucradas en inves- tigación y/o transferencia de tecnología en este cultivo en Costa Rica, además de intercambio de conocimientos y tecnología con países de Centroamérica y El Caribe, México y con el Centro Internacional de Agricultura Tropical (CIAT). Una de las prioridades establecidas por el PITTA-Frijol a partir del estableci-

\footnotetext{
1 Investigación financiada parcialmente por el Programa Cooperativo Regional de Frijol para Centroamérica, México y El Caribe (PROFRIJOL)

2 Dirección de Investigaciones Agrícolas, Ministerio de Agricultura y Ganadería, San José Costa Rica. E-mail: jhernandez@ns.mag.go.cr

3 Estación Experimental Fabio Baudrit Moreno, Universidad de Costa Rica (Proyecto 736-91-315 Vicerrectoría de Investigación). Apartado postal 183-4050 Alajuela Costa Rica. E-mail: avillalo@cariari.ucr.ac.cr.

4 Dirección de Investigaciones Agrícolas, Ministerio de Agricultura y Ganadería, San José Costa Rica. E-mail: amorales@ns.mag.go.cr.
} 
miento del Tratado de Libre Comercio en 1995, fue la obtención de variedades de color de grano rojo brillante, debido a su mayor precio de mercado y posibilidad de exportación de excedentes a Centroamérica .

A partir de 1996 el programa de mejoramiento dio un mayor énfasis a los viveros que contenían material rojo, procedente de la Red PROFRIJOL (Programa Regional de Frijol para Centroamérica, México y El Caribe).

El frijol negro pequeño ha sido el principal grano de consumo en Costa Rica con más de $50 \%$ del total de la ingesta anual, mientras que el grano rojo representa cerca del $20 \%$ y el $30 \%$ restante le es indiferente el color del grano. Esta preferencia fue inducida por la política de precios similares para ambos tipos de frijol por parte del Estado, lo cual llevó a los agricultores a producir variedades de color negro, ya que genéticamente producen mayor rendimiento por área, no obstante, la mayor oferta de frijol negro en un mercado que no ofrecía alternativas, acabó por formar una preferencia por costumbre (Salazar, 1999).

Con las nuevas políticas gubernamentales, el Consejo Nacional de Producción salió del área de fijación de precios en los granos básicos y del comercio de la compra y venta de granos. Esto provocó una liberación de precios que afectó considerablemente el precio del frijol rojo y negro, el grano rojo tomó más valor debido a una menor oferta y ello ha provocado un aumento en la demanda de variedades de grano rojo.

\section{MATERIALES Y MÉTODOS}

La variedad Bribri proviene de la línea MD 23-24. Esta línea fue desarrollada por el Programa de mejoramiento genético de Frijol del Dr. Juan Carlos Rosas de la Escuela Agrícola Panamericana (EAP), Zamorano, Honduras e introducida a Costa Rica en 1996 por el (PITTA-Frijol). La línea MD 2324 se originó de la cruza (RAB 310/XAN 155) x (DOR 391/POMPADOUR G). El origen de los progenitores es: RAB 310= DOR 364 x (SEL 277/BAT 1514); XAN 155= BAT 930/BAT 93; DOR 391= DOR 367 x (DOR $364 /$ IN 101); y POMPADOUR G= variedad criolla de la República Dominicana.

\section{Características de las regiones donde se evaluó la variedad Bribri}

Región Huetar Norte: La zona de mayor área de siembra de frijol en Costa Rica es la Región Huetar
Norte. Las áreas de cultivo de esta leguminosa varían desde 10 ha, hasta grandes explotaciones de más de 50 ha. Predominan suelos llanos y ondulados, moderadamente profundos de textura fina, presentan un buen drenaje, pero en su mayoría son de baja fertilidad. En el cantón de Los Chiles predominan los Ultisoles de muy baja fertilidad, $\mathrm{pH}$ inferior a 5,5 y alta acidez: Typic Hapludult y Typic Haplohumult. En los cantones de Upala y Guatuso hay suelos Ultisoles e Inceptisoles y algunas asociaciones de origen volcánico: Andic Humitropept, Typic Hapludult y Typic Dystropept. Todos ellos con bajo contenidos de bases, $\mathrm{pH}$ entre 5,0 y 5,8 y colores pardo oscuros a pardo grisáceos, presentan una fertilidad más alta que los de la zona de Los Chiles y predominan los pequeños agricultores.

Región Brunca: Las zonas frijoleras cuentan con áreas de topografía accidentada fuertemente ondulada y quebrada, suelos de buena a moderada profundidad, con texturas finas, moderada permeabilidad y muy propensos a la erosión. Son de baja fertilidad y en algunos casos se presenta pedregosidad. Los suelos se clasifican en Ultisoles en su mayoría, Inceptisoles y Entisoles. Hacia la región de Chánguena se presentan suelos de reciente formación y con buen contenido de materia orgánica. Se les clasifica como: Ustic Dystropept asociado con Typic Haplustalf y Typic-Lythic Usthorthent para las localidades de Chánguena y Guadalupe y Typic Haplustult asociado con Ustic Humitropept en el cantón de Buenos Aires y localidades de Veracruz y Concepción. En esta región predominan pequeños agricultores (2-5 ha) con rotación de cultivos maíz-arroz-frijol.

Región Central: En el cantón de Puriscal de la Región Central, se cultiva frijol a escala reducida en comparación con las anteriores regiones. Los suelos son escarpados a fuertemente ondulados, de texturas finas, con buen drenaje y poco permeables. Su fertilidad es de media a baja. Son suelos Ultisoles y se les clasifica como Ustic Haplohumult con asociaciones de Typic Rhodustult, Typic Dystrandept y Typic Ustorthent. Estos suelos presentan bajos contenidos de materia orgánica y problemas de fuerte erosión. La tenencia de la tierra es de pequeños agricultores (2-5 ha).

\section{Representación gráfica de las localidades donde se evaluó la variedad Bribri en Costa Rica}

En la Figura 1, se indican los sitios donde se evaluó la variedad Bribri. Estos sitios son los más representativos en la producción comercial de frijol con base en la zonificación agrícola y el aporte porcentual de éstas en la producción de frijol (Araya y Araya, 1996). 


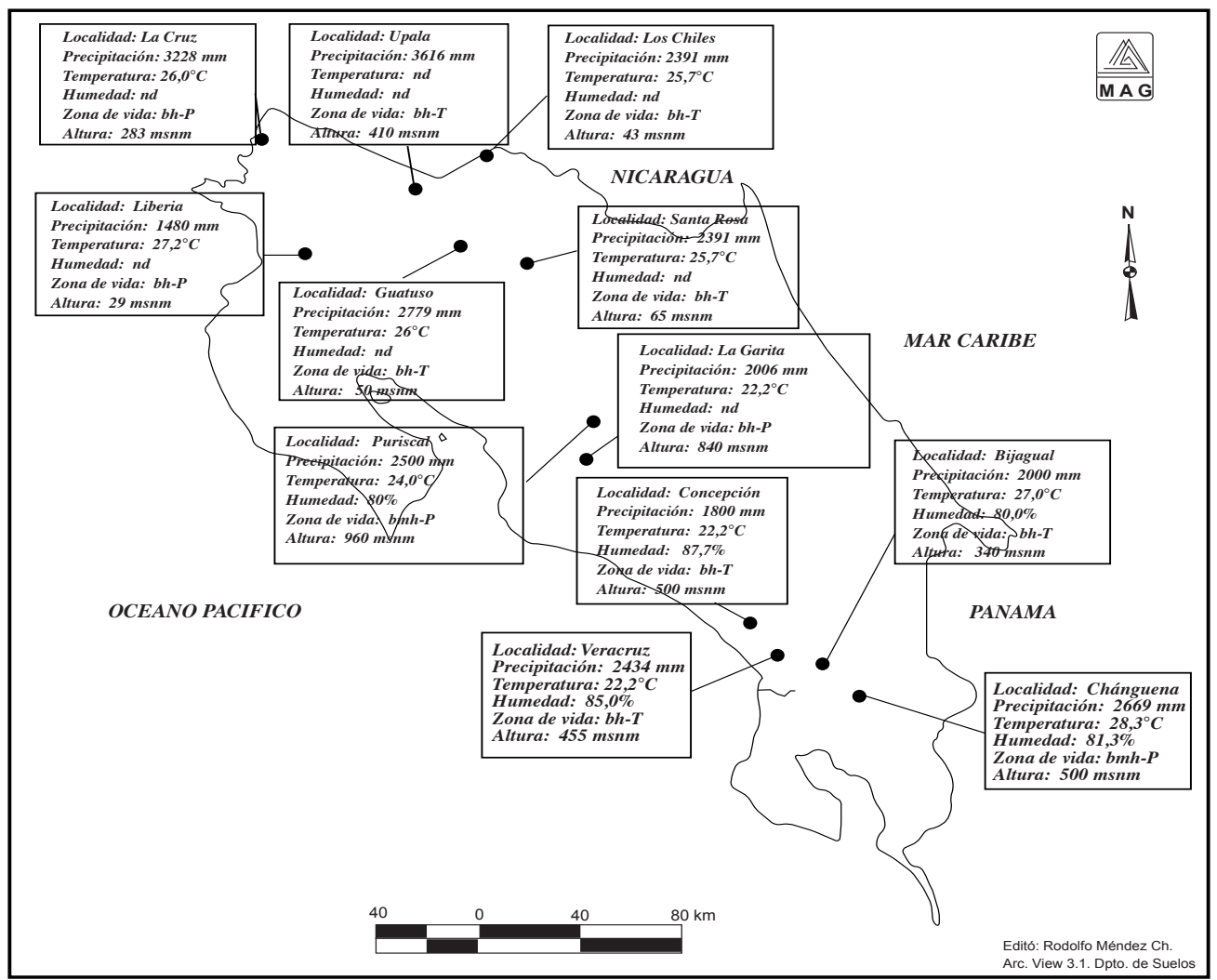

Figura 1. Costa Rica: Localidades y condiciones climáticas donde se evaluó la variedad de frijol Bribri.

Las altitudes de las localidades varían desde 43 hasta 960 msnm, lo que cubre todo el rango donde se efectuan las siembras comerciales de frijol. Las zonas de vida donde se cultiva más el frijol son la bh-T (bosque húmedo tropical) y el bmh-P (bosque muy húmedo Premontano), siendo la primera la más importante en área de siembra comercial. En las localidades ubicadas en esta zona de vida la temperatura varía entre 22 y $27^{\circ} \mathrm{C}$ y la lluvia entre 1800 y $3600 \mathrm{~mm}$ anuales.

\section{Evaluación experimental de la variedad Bribri}

Las evaluaciones a través del tiempo y localidades de la variedad Bribri, fueron realizadas con base en el Ensayo Centroamericano de Adaptación y Rendimiento (ECAR), Ensayo Nacional de Adaptación y Rendimiento (ENAR), el Vivero Histórico Nacional (vivero con las variedades nacionales, líneas promisoarias y variedades criollas) y el Vivero F-6 y F-7 para Altas Temperaturas (Vivero procedente de la Escuela Agrícola Panamericana El Zamorano), ademas de las Parcelas de Verificación y de Validación. A continuación se describen cada uno de los ensayos:

\section{A.) Ensayo Centroamericano de Adaptación y Ren- dimiento (ECAR), período 1996-1998}

El experimento denominado Ensayo Centroamericano de Adaptación y Rendimiento de Grano Rojo (ECAR-ROJO), compuesto por 16 genotipos y proveniente de la EAP fue establecido en 1996 en los Los Chiles y Upala, y en 1997 y 1998 en Los Chiles y Puriscal. Los tratamientos se presentan en el Cuadro 1.

\section{B.) Ensayo Nacional de Adaptación y Rendimiento (ENAR). Período 1997 y 1998.}

Durante los años 1995 y 1996 con base en la evaluación de diferentes viveros (ECAR, VIDAC (Vivero de Adaptación Centroamericano, y Viveros Específicos), evaluados por diferentes investigadores del PITTA-Frijol, fueron identificados y seleccionados los cultivares superiores de grano rojo y negro. Con los 12 mejores genotipos más cuatro testigos, fue creado el Ensayo Nacional de Adaptación y Rendimiento (ENAR), para estudiar el comportamiento agronómico y estabilidad en los rendimientos de los materiales en las principales zonas frijoleras del país (Cuadro 2). El 
Cuadro 1. Identificación y procedencia de las líneas de frijol del Ensayo Centroamericano de Adaptación y Rendimiento (ECAR), evaluadas entre 1996 y 1998 en Costa Rica.

\begin{tabular}{|c|c|c|c|c|c|}
\hline \multirow[b]{2}{*}{$\#$} & \multicolumn{2}{|c|}{ 1996-1997 } & \multicolumn{3}{|c|}{1998} \\
\hline & Genotipo & Programa & $\#$ & Genotipo & Programa \\
\hline 1 & DORADO & CIAT Guatemala & 1 & PCE 9351-8 & EAP Honduras \\
\hline 2 & DOR 472 & CIAT Guatemala & 2 & MD 2324 (Bribri) & EAP Honduras \\
\hline 3 & DOR 481 & CIAT Guatemala & 3 & DOR 364 & CIAT Guatemala \\
\hline 4 & DOR 482 & CIAT Guatemala & 4 & SRC $1-2-12$ & EAP Honduras \\
\hline 5 & DOR 483 & CIAT Guatemala & 5 & DOR 557 & CIAT Guatemala \\
\hline 6 & DOR 484 & CIAT Guatemala & 6 & DICTA 122 & DICTA Honduras \\
\hline 7 & DOR 488 & CIAT Guatemala & 7 & $9609-2-2$ & EAP Honduras \\
\hline 8 & DOR 513 & CIAT Guatemala & 8 & DICTA 146 & DICTA Honduras \\
\hline 9 & RAB 478 & CIAT Colombia & 9 & DOR 576 & CIAT Guatemala \\
\hline 10 & MD 3018 & EAP Honduras & 10 & ICTA JU 95-4 & CIAT Guatemala \\
\hline 11 & MD 3019 & EAP Honduras & 11 & SRC 1-18-1 (A) & EAP Honduras \\
\hline 12 & MD 2324 (Bribri) & EAP Honduras & 12 & SRC 1-1-18 & EAP Honduras \\
\hline 13 & MD 3097 & EAP Honduras & 13 & $9609-16-3$ & EAP Honduras \\
\hline 14 & DICTA 113 & DICTA Honduras & 14 & SRC 1-12-1 & EAP Honduras \\
\hline 15 & DICTA 122 & DICTA Honduras & 15 & PM 9422-3 & EAP Honduras \\
\hline 16 & Testigo Local & & 16 & Testigo local & \\
\hline
\end{tabular}

*/Variedad Bribri

objetivo, fue identificar los cultivares mejor adaptados, más productivos, con mejor comportamiento agronómico y tolerantes a los principales problemas fitopatológicos. Los mejores cultivares identificados de éstos ensayos son incluídos en las parcelas de verificación y validación a nivel de finca. Fueron estudiados 12 cultivares en 1997 y ocho en 1998, comparados con los testigos nacionales Guaymi (MUS 106) de grano negro y

Cuadro 2. Identificación, procedencia y color grano de las líneas de frijol del Ensayo Nacional de Adaptación y Rendimiento (ENAR) evaluadas entre 1997 y 1998 en Costa Rica.

\begin{tabular}{|c|c|c|c|c|}
\hline$\#$ & 1997 & Proced & dencia & \\
\hline 1 & DOR-808 & & CIAT-Guatemala & Rojo \\
\hline 2 & DOR-802 & DOR-802 & CIAT-Guatemala & Rojo \\
\hline 3 & DOR-812 & & CIAT-Guatemala & Rojo \\
\hline 4 & MD 2324 & MD 2324 & EAP-Honduras & Rojo \\
\hline 5 & DOR-483 & & CIAT-Guatemala & Rojo \\
\hline 6 & MD-30-97 & MD-30-97 & EAP-Honduras & Rojo \\
\hline 7 & MD-30-19 & MD-30-19 & EAP-Honduras & Rojo \\
\hline 8 & UCR-54 & & PNF*-Costa Rica & Rojo \\
\hline 9 & UCR-19 & & PNF-Costa Rica & Rojo \\
\hline 10 & ICTA-JU-95-42 & & CIAT-Guatemala & Negro \\
\hline 11 & ICTA-JU-95-91 & ICTA-JU-95-91 & CIAT-Guatemala & Negro \\
\hline 12 & UCR-55 & UCR-55 & PNF-Costa Rica & Negro \\
\hline 13 & Chirripó rojo*** & Chirripó rojo**** & CIAT-Colombia & Rojo \\
\hline 14 & Guaymi $* * *$ & Guaymi $* * *$ & CIAT-Colombia & Negro \\
\hline 15 & Testigo Local Rojo & Testigo Local Rojo & $\begin{array}{l}\text { Var. Adoptada o } \\
\text { criolla local }\end{array}$ & Rojo \\
\hline 16 & Testigo Local Negro & o Testigo Local Negro & $\begin{array}{l}\text { Var. Adoptada o } \\
\text { criolla local }\end{array}$ & Negro \\
\hline & SRC 1-12-1 & SRC 1-12-1 & EAP-Honduras & Rojo \\
\hline & BAC $50 * *$ & BAC 50 & Brasil & Rojo \\
\hline
\end{tabular}

*PNF= Líneas del Programa Nacional de Frijol de Costa Rica. **Sustituído por DOR 576 en la segunda época de siembra de 1998. *** Testigo nacional
Chirripó Rojo (DOR 489-S) de grano de color rojo y los respectivos testigos locales de grano rojo y negro (Araya et al., 1998; Morales, 1998; Morales et al., 1998).

En total fueron establecidos 17 ensayos ENAR a través de las épocas y localidades.

\section{C.) Evaluación de resistencia a patógenos}

La Estación Experimental Fabio Baudrit ubicada en La Garita de Alajuela a 840 msnm y la Sub Estación Fraijanes a $1650 \mathrm{msnm}$, se evaluaron los materiales por su resistencia a diferentes patógenos así como su respuesta a suelos de baja fertilidad. La metodología incluye la inoculación con antracnosis y se da incidencia natural de roya, Chaetoseptoria sp., mancha angular y mosaico dorado.

\section{Parcelas de Verificación}

Región Brunca: En las localidades de Chánguena, Concepción, Veracruz y Sabana, se verificó el comportamiento de los cultivares promisorios de color rojo MD 2324 (Bribri) y MD 3019, comparados con los respectivos testigos locales de grano rojo. Éstos cultivares fueron seleccionados con base en su rendimiento, características comerciales y su comportamiento agronómico. Las parcelas fueron establecidas bajo el manejo convencional de los productores (Araya et al., 1999). Se entregó a los agricultores un kilogramo de semilla de cada uno de los cultivares. El área sembrada varió entre 300 y $450 \mathrm{~m}^{2}$ por kilo de semilla. 
Región Huetar Norte: Se establecieron seis parcelas de verificación en la Región Huetar Norte. Los cultivares evaluados fueron MUS 181 de grano negro y MD 2324 (Bribri) de color rojo, cada uno de ellos comparados con los respectivos testigos locales de color rojo o negro. Cada agricultor empleó el sistema de siembra y manejo agronómico que tradicionalmente utiliza en su finca. Las parcelas constaron de veinte hileras de frijol de $10 \mathrm{~m}$ de longitud, separadas entre 0,6 y $0,5 \mathrm{~m}$ entre surcos (Araya et al., 1999).

\section{Parcelas de Validación}

La línea MD 2324 (Bribri) se validó en once localidades y comparó con la variedad local. En la Región Brunca se establecieron 18 parcelas, se distribuyeron cinco kilos de semilla de cada cultivar por agricultor en las localidades de Veracruz, Concepción, La Sabana, Chánguena y Potrero Grande. En la Región Huetar Norte se establecieron cuatro parcelas. Se entregaron $10 \mathrm{~kg}$ de semilla en las localidades de Guatuso y Upala y $32 \mathrm{~kg}$ en Los Chiles y Santa Rosa. Cada parcela varió de tamaño con base en el sistema de siembra de cada agricultor. (Araya et al., 2000; Hernández et al., 2000).

\section{Análisis de Resultados}

El análisis de los resultados se basó en la recopilación de la información obtenida en las investigaciones efectuadas en ensayos, viveros y parcelas de verificación y validación. Se procedió a su tabulación e interpretación con base en Regiones, rendimiento de los testigos y promedio general de producción de grano, así como incidencia de patógenos.

Algunas de las características del comportamiento agronómico de la variedad Bribri, así como de sus características organolépticas, fueron producto de la observación de los agricultores involucrados.

\section{RESULTADOS}

\section{Análisis del comportamiento de la variedad Bribri en los ensayos y viveros}

En el Cuadro 3 se observa el rendimiento en $\mathrm{kg} / \mathrm{ha}$ de la variedad Bribri en 25 ensayos establecidos en cuatro regiones frijoleras (Mora et al., 1997; Morales, 1998; Araya et al., 1998). El análisis general de los datos mostró que en el 68\% de los ensayos, la Bribri superó en rendimiento al testigo local, en un $50 \%$ al testigo nacional y en el restante $48 \%$ de las evaluacio- nes tuvo una producción similar a la média general de todos los ensayos.

La variedad Bribri mostró una posición intermedia en rendimiento en grano, lo que indica que su potencial comercial no sólo está basado en su rendimiento, sino también por su reacción de tolerancia a la enfermedades principalmente la mustia hilachosa (Cuadro 4).

La variedad Bribri superó en rendimiento promedio, al testigo nacional en $17,2 \%$ y al testigo local en $13,4 \%$. Su producción fue superior en un $8,5 \%$ al promedio de rendimiento obtenido en todos los ensayos.

En el Cuadro 3 se observa que Bribri mostró un alto rendimiento $(1470 \mathrm{~kg} / \mathrm{ha})$ y una buena adaptación en la localidad de Liberia, Guanacaste. La Región Chorotega posee además condiciones para siembras bajo riego y en rotación con arroz, lo que sugiere su uso potencial como alternativa para esta Región.

En relación con las dos principales zonas frijoleras, el mayor rendimiento de la variedad Bribri se dió en la Región Huetar Norte. En esta zona hay una menor presión de patógenos (Cuadro 4) debido a que sólo se da una sola época de siembra de frijol. Además de suelos menos erosionados por presentar esta región una topografía entre llana y ondulada.

La mayor productividad que obtuvo Bribri a través de todas las localidades y épocas fue de $1811 \mathrm{~kg} / \mathrm{ha}$, en El Parque, sitio ubicado en la localidad de Los Chiles, mientras que el rendimiento más bajo se presentó en Tujankir de Guatuso con 368 kg/ha. Su producción promedio fue de $988 \mathrm{~kg} / \mathrm{ha}$.

La Bribri mostró tolerancia a la mustia hilachosa (Cuadro 4) y ésta fue la principal ventaja en resistencia a patógenos de esta variedad en relación con el testigo local. La tolerancia a la mustia hilachosa de la variedad Bribri, pudo estar influido por su hábito tipo II A (planta erecta con poca guía) factor que contribuyó al combate integral de este patógeno ya que complementó el empleo de coberturas y funguicidas. Esto influyó además en que los rendimientos en la etapa de validación fueron superiores a los obtenidos en los ensayos donde no hubo protección agroquímica.

\section{Parcelas de Verificación de la variedad Bribri}

Región Brunca: Los resultados obtenidos en las parcelas establecidas bajo el manejo convencional de los productores se muestra en el Cuadro 5 . 
Cuadro 3. Rendimiento en kg/ha de la variedad Bribri desde su introducción a Costa Rica, por Región, localidad y año de evaluación.

\begin{tabular}{|c|c|c|c|c|c|c|c|c|}
\hline \multirow[t]{2}{*}{$\#$} & \multirow[t]{2}{*}{ Localidad } & \multirow[t]{2}{*}{ Año } & \multirow[t]{2}{*}{ Tipo Ensayo } & \multicolumn{3}{|c|}{ Rendimiento (kg/ha) } & \multirow{2}{*}{$\begin{array}{l}\text { Promedio/ } \\
\text { Ensayo }\end{array}$} & \multirow{2}{*}{$\begin{array}{l}\text { Posición } \\
\text { Bribri** }\end{array}$} \\
\hline & & & & Bribri & $\begin{array}{l}\text { Testigo } \\
\text { Local }\end{array}$ & $\begin{array}{c}\text { Testigo } \\
\text { Nacional* }^{*}\end{array}$ & & \\
\hline \multicolumn{9}{|c|}{ Región Huetar Norte } \\
\hline 1 & Upala & $96 \mathrm{~B}$ & ECAR & 1539 & 1415 & -- & 1325 & 2 de 16 \\
\hline 2 & Los Chiles & $96 \mathrm{~B}$ & ECAR & 802 & 654 & -- & 669 & $11 \mathrm{de} 16$ \\
\hline 3 & Tujankir & $97 \mathrm{~B}$ & ENAR & 368 & 431 & 697 & 564 & 16 de 16 \\
\hline 4 & La Vega & $97 \mathrm{~B}$ & ENAR & 1512 & 1130 & 1290 & 1238 & 4 de 16 \\
\hline 5 & Bijagua & $97 \mathrm{~B}$ & ENAR & 528 & 331 & 633 & 535 & 10 de 16 \\
\hline 6 & Los Chiles & $98 \mathrm{~B}$ & ENAR & 1310 & 1161 & 941 & 1178 & 4 de 12 \\
\hline 7 & Los Chiles & 98B & ECAR & 1811 & 842 & -- & 1473 & 3 de 16 \\
\hline \multicolumn{4}{|c|}{ Promedio } & 1124 & 852 & 890 & 997 & \\
\hline \multicolumn{9}{|c|}{ Región Brunca } \\
\hline 1 & Veracruz & $97 \mathrm{~A}$ & ENAR & 582 & 951 & 703 & 663 & 10 de 16 \\
\hline 2 & Concepción & $97 \mathrm{~A}$ & ENAR & 1215 & 1955 & 1707 & 1524 & 15 de 16 \\
\hline 3 & Concepción & $97 \mathrm{~B}$ & ENAR & 669 & 736 & 676 & 619 & 6 de 16 \\
\hline 4 & La Sabana & $97 \mathrm{~B}$ & ENAR & 1047 & 663 & 725 & 872 & 2 de 16 \\
\hline 5 & Changuena & $97 \mathrm{~B}$ & ENAR & 1200 & 623 & 714 & 713 & $1 \mathrm{de} 16$ \\
\hline 6 & Concepción & $98 \mathrm{~A}$ & ENAR & 896 & 1159 & 1232 & 1094 & 10 de 12 \\
\hline 7 & Veracruz & $98 \mathrm{~A}$ & ENAR & 934 & 255 & 322 & 623 & 1 de 12 \\
\hline 8 & Concepción & $98 \mathrm{~B}$ & ENAR & 695 & 675 & 764 & 910 & 11 de 12 \\
\hline 9 & La Sabana & $98 \mathrm{~B}$ & ENAR & 1248 & 1153 & 1026 & 1355 & 8 de 12 \\
\hline \multicolumn{4}{|c|}{ Promedio } & 942 & 908 & 874 & 930 & \\
\hline \multicolumn{9}{|c|}{ Región Central } \\
\hline 1 & La Legua & $97 \mathrm{~A}$ & ENAR & 1291 & 1165 & 1301 & 1328 & 10 de 16 \\
\hline 2 & Puriscal & $97 \mathrm{~B}$ & ENAR & 696 & 356 & 617 & 676 & 7 de 16 \\
\hline 3 & Puriscal & $97 \mathrm{~B}$ & ECAR & 844 & -- & 642 & 789 & 7 de 16 \\
\hline 4 & Puriscal & $98 \mathrm{~A}$ & ENAR & 677 & 963 & 791 & 762 & 7 de 12 \\
\hline 5 & Puriscal & $98 \mathrm{~B}$ & ENAR & 746 & 1028 & 399 & 796 & 8 de 12 \\
\hline 6 & Puriscal & 98B & ECAR & 563 & 442 & -- & 411 & 2 de 16 \\
\hline 7 & Alajuela & $98 \mathrm{~B}$ & Vivero & 590 & 1145 & -- & 833 & 21 de 33 \\
\hline & & & Histórico & & & & & \\
\hline \multicolumn{4}{|c|}{ Promedio } & 772 & 850 & 750 & 799 & \\
\hline \multicolumn{9}{|c|}{ Región Chorotega } \\
\hline 1 & Liberia & $98 \mathrm{~B}$ & $\begin{array}{l}\text { Alta } \\
\text { Temperatura }\end{array}$ & 1470 & 850 & -- & ND & 18 de 114 \\
\hline 2 & Liberia & $98 \mathrm{~B}$ & $\begin{array}{l}\text { Alta } \\
\text { Temperatura }\end{array}$ & 1470 & 840 & -- & ND & 11de 104 \\
\hline \multicolumn{4}{|c|}{ Promedio } & 1470 & 845 & -- & & \\
\hline \multicolumn{4}{|c|}{ Promedio General } & 988 & 871 & 843 & 911 & \\
\hline
\end{tabular}

*/Testigo Chirripó Rojo.

**/Posición referida a su rendimiento en orden ascendente con respecto al total de cultivares evaluados.

Cuadro 4. Reacción de la variedad Bribri a las principales enfermedades por Región. Costa Rica.

\begin{tabular}{llccccccccccccc}
\hline \multirow{2}{*}{ Región } & \multicolumn{2}{c}{ AMA } & \multicolumn{2}{c}{ ROYA* } & \multicolumn{3}{c}{ ANT* } & MUS* & \multicolumn{3}{c}{ ALS* } & \multicolumn{3}{c}{ Chaetoseptoria w } \\
& & V** & T** & V & T & V & T & & & V & T & V & T \\
\hline 1 & Central & - & - & 6 & 4 & 6 & 7 & 4 & 8 & 4 & 5 & 6 & 6 \\
2 & Brunca & 6 & 7 & - & - & 4 & 5 & 5 & 7 & 6 & 7 & - & - \\
3 & Huetar Norte & - & - & - & - & - & - & - & - & 4 & 4 & - & - \\
\hline
\end{tabular}

* AMA= Amachamiento, causado por el virus del moteado clorótico del Cowpea. ANT=antracnosis. MUS$=$ mustia hilachosa. ALS= mancha angular.

** V=Bribri. $\mathrm{T}=$ testigo.

Bribri mostró buen comportamiento en Upala, Los Chiles y Santa Rosa localidades de la Región Huetar Norte y en Concepción y Veracruz ubicadas en la zona frijolera de la Región Brunca (Cuadro 5). Asimismo, superó a los testigos locales en un $67 \%$ de las evaluaciones y aventajó a la línea MD 3019 en dos de los tres en- 
Cuadro 5. Rendimiento en $\mathrm{kg} / \mathrm{ha}$ de las parcelas de verificación de la variedad Bribri, en Costa Rica, 1998B.

\begin{tabular}{llccc}
\hline$\#$ & Localidad & Bribri & MD 3019 & Testigo Local \\
\hline 1 & Upala & 1071 & -- & 500 \\
2 & Los Chiles & 1208 & -- & 750 \\
3 & Santa Rosa & 1800 & -- & 1600 \\
4 & Concepción & 1562 & 1562 & 1875 \\
5 & Bijagua & 580 & -- & 450 \\
6 & Veracruz & 1665 & 1140 & 1440 \\
7 & Guatuso & 575 & -- & 625 \\
8 & Guatuso & 325 & -- & 490 \\
9 & Changuena & 333 & 233 & 166 \\
& Promedio & 1013 & 978 & 877 \\
\hline
\end{tabular}

sayos donde se incluyó este material además de mostrar una arquitectura más erecta que la MD 3019. De acuerdo con la média general, Bribri rindió $1013 \mathrm{~kg} / \mathrm{ha}$ y superó en un 3,6\% a MD 3019 y 13,4 \% al grupo de testigos. Esto evidenció su potencial de rendimiento, valor comercial y justificó su paso a las pruebas de validación.

Una vez concluída esta fase, los productores mostraron satisfacción por el rendimiento obtenido, comportamiento agronómico apropiado a sus sistemas de producción e interés por probar este material en parcelas comerciales.

\section{Validación de la variedad Bribri}

La validación del cultivar fue realizada en 16 localidades y durante dos ciclos de siembra en las Regiones Brunca y Huetar Norte.

Con base en los análisis de suelos de los sitios estudiados, se agruparon las localidades de acuerdo con el nivel de fertilidad en tres categorías: suelos de alta fertilidad, media fertilidad y de baja fertilidad (Corella, 1995) (Cuadro 6). Se observó en los terrenos de baja fertilidad que la productividad de la variedad Bribri fue superior con respecto al testigo, pero cuando se estableció en suelos de alta y mediana fertilidad, los rendimientos fueron similares al testigo, en particular cuando se empleó como testigo el Sacapobres rojo. Esto podría influir en la reducción de los costos de producción por un menor empleo de fertilizantes, además de que favorece a los agricultores con pocos recursos y en terrenos de baja fertilidad. Implica además un potencial de manejo más amigable con el medio ambiente. Ademas por lo general los productores de frijol usan bajos insumos y sus cultivos se localizan en suelos erosionados, marginales y de baja fertilidad.

En finca de agricultores su productividad fue de $972 \mathrm{~kg} / \mathrm{ha}$ con un máximo de $1930 \mathrm{~kg} / \mathrm{ha}$. Con base en el promedio general de rendimiento, la variedad Bribri superó al testigo local en el $80 \%$ de las evaluaciones.

Los agricultores participantes en la validación consideraron a Bribri para uso comercial, basados en su tolerancia a mustia hilachosa, su arquitectura y color de grano. Manifestaron la importancia de incluirlo en el programa de producción local de semilla.

\section{Otras características agronómicas y organolépticas observadas en la variedad Bribri}

La variedad Bribri es de hábito de crecimiento arbustivo Tipo II-B, de porte erecto y ciclo vegetativo entre 76 y 80 días a cosecha. El periodo de floración varió entre 38 y 41 días después de la siembra. La madurez fisiológica se dió entre los 68 y 72 días después de la siembra, el color de tallo es verde en la fase vegetativa y rosado en la etapa de madurez fisiológica. El color de vainas es verde durante la etapa de llenado de grano y rosadas de la mitad de la valva hacia el dorso, en la etapa de madurez fisiológica. El peso de 100 semillas varió entre 18 y 20 gramos y su color es rojo oscuro brillante, estable bajo diversas localidades, épocas de siembra y almacenamiento y de buena aceptación comercial lo que indica su potencial de comercialización. Posee buen sabor de grano, caldo denso y un corto tiempo de cocción.

Cuadro 6. Producción de las parcelas de validación de la variedad Bibri y el testigo local ( $\mathrm{kg} / \mathrm{ha})$, con base en el nivel de fertilidad de los suelos. Costa Rica, 1999.

\begin{tabular}{|c|c|c|c|c|}
\hline$\#$ & Localidad & Año & Bribri & Testigo Local \\
\hline \multicolumn{5}{|c|}{ Suelos de alta fertilidad } \\
\hline 1 & Concepción & $99 \mathrm{~A}$ & 507 & 500 \\
\hline 2 & Concepción & $99 \mathrm{~B}$ & 1479 & --- \\
\hline 3 & Concepción & $99 \mathrm{~B}$ & 1533 & 1500 \\
\hline 4 & Potrero Grande & $99 \mathrm{~B}$ & 1375 & 1860 \\
\hline 5 & Changuena & $99 \mathrm{~B}$ & 1600 & 1600 \\
\hline \multicolumn{3}{|c|}{ Promedio } & 1299 & 1378 \\
\hline \multicolumn{5}{|c|}{ Suelos de mediana fertilidad } \\
\hline 6 & Guatuso & $99 \mathrm{~B}$ & 354 & ---- \\
\hline 7 & Veracruz & $99 \mathrm{~A}$ & 1462 & 1380 \\
\hline 8 & Santa Rosa & $99 \mathrm{~B}$ & 538 & 460 \\
\hline 9 & El Parque & $99 \mathrm{~B}$ & 598 & ---- \\
\hline 10 & Llano Azul & 99B & 552 & ---- \\
\hline 11 & Potrero Grande & $99 \mathrm{~B}$ & 927 & 891 \\
\hline 12 & Veracruz & $99 \mathrm{~B}$ & 1930 & 1380 \\
\hline \multicolumn{3}{|c|}{ Promedio } & 909 & 1028 \\
\hline \multicolumn{5}{|c|}{ Suelos de baja fertilidad } \\
\hline 13 & Veracruz & $99 \mathrm{~B}$ & 390 & 138 \\
\hline 14 & La Sabana & $99 \mathrm{~B}$ & 660 & 360 \\
\hline 15 & La Sabana & $99 \mathrm{~B}$ & 501 & 393 \\
\hline & Changuena & $99 \mathrm{~B}$ & 1150 & 383 \\
\hline \multicolumn{3}{|c|}{ Promedio } & 675 & 318 \\
\hline \multicolumn{3}{|c|}{ Promedio General } & 972 & 904 \\
\hline
\end{tabular}


La decoloración del grano es una característica no deseable y muy frecuente en variedades de grano rojo. La estabilidad en el color del grano de la variedad Bribri bajo diferentes condiciones de manejo, localidades, épocas de siembra y almacenamiento, le han conferido una gran aceptación por parte del agricultor y los comerciantes.

Los agricultores indicaron como aspecto relevante que la variedad Bribri es fácil de trillar. Los cultivares que presentan dificultad de desgrane debido a la dureza de las valvas tienen problemas de adopción entre pequeños y grandes productores de frijol. Además indicaron que mostró poco acame (lo que fue atribuido al grosor de su tallo), considerado una característica deseable.

El valor agronómico integra en un solo valor las diferentes característica favorables o desfavorables presentes en una línea o variedad. Incluye tipo de planta, sanidad y potencial de rendimiento. La variedad Bribri posee porte erecto, tolerancia a la mustia hilachosa, con una buena productividad en baja fertilidad. Estas características la hacen adecuada para los sistemas de siembra de frijol.

En la Región Brunca, en particular en las localidades de Concepción de Pilas y Veracruz de Pejibaye, se sembraron en mayo de 2000 alrededor de 100 ha con semilla de la variedad Bribri. Los agricultores se dedicaron a incrementarla, debido a las ventajas que fueron observando en los ensayos, en los cuales ellos participaron. Esta adopción temprana, es el principal indicativo del potencial de esta variedad.

\section{AGRADECIMIENTO}

Los autores desean constar que en la obtención de una variedad intervienen gran cantidad de personas, cuyo trabajo es de gran relevancia para asegurar la selección de una línea para uso comercial. Las personas descritas a continuación formaron parte básica de la selección de la variedad Bribri: Ministerio de Agricultura y Ganadería: Bernardo Mora Brenes, Santiago Fernández, Miguel Acosta Maroto, Santana Jiménez Solís, Roberto Chacón Montero, Mario Montero Mayorga, William Pizarro Galeano, Arnoldo Vargas León, Pablo Murillo Murillo, Carlos Xatruch Corrales, Olman Villegas, Pedro Martínez Álvarez, Carlos Cordero Morales, Omar Campos Duarte, Roger Campos Barboza, Arturo Solórzano Arroyo, Eduardo Elizondo Chinchilla. Universidad Nacional: Carlos Araya Fernández. Instituto Tecnológico de Costa Rica: Gerardo Chaves. Consejo Nacional de Producción: José Joaquín Salazar Rojas, Ned Gordon Zamora.
Oficina Nacional de Semillas: Orlando Carrillo. Agricultores: Juan Morera Cortez, Talí Bermúdez Camacho, Mario Morera Cortéz, Adalberto Morera Cortez, Victor Fallas Chinchilla, Ramón Fallas Chinchilla, Cruz Elizondo, Alberto Elizondo, Eduardo Mena Arias, Rodrigo Loría, Omar Porras, Ronald Campos, Victorino Campos, Jose Irigoyen, Lisandro Reyes, Alfredo Montoya, Carlos Orozco, Andres Potoy, Roger Murillo, Delfi Cabrera, Ernestino Quezada, Leonidas Correa, Ramón Delgado y Steven Polen.

\section{LITERATURA CITADA}

ARAYA, R; ARAYA, C. 1996. Guía para el establecimiento, manejo y evaluación del Vivero Preliminar Nacional (VPN), Ensayo Nacional de Adaptación y Rendimiento (ENAR) y validación de líneas promisorias, del Programa de Investigación de Tecnología Agropecuaria Frijol Costa Rica (PITTA-Frijol). San José, Costa Rica. 23 p.

ARAYA, C. M.; ARAYA, R.; MORALES, A.; ACUÑA, O.; FLORES G. 1998. Informe técnico anual POA 97-98. Programa de investigación y transferencia de tecnología agropecuaria-frijol (PITTA-Frijol). San José, Costa Rica. 33 p.

ARAYA, C. M.; ARAYA, R.; MORALES, A.; ACUÑA, O.; FLORES G. 1999. Informe técnico anual POA 98-99. Programa de investigación y transferencia de tecnología agropecuaria-frijol (PITTA-Frijol). San José, Costa Rica. 53 p.

ARAYA, C. M.; ARAYA, R.; HERNÁNDEZ, J. C.; BLANCO, H. 2000. Informe técnico anual POA 99-2000. Programa de Investigación y Transferencia de Tecnología Agropecuaria-Frijol (PITTA-Frijol). San José, Costa Rica. 44 p.

CORDERO, C.; MORALES, A. 1999. Evaluación del Ensayo Centroamericano de Adaptación y rendimiento (ECAR-Rojo) El Estero, Puriscal 1998. In: Tercer taller anual de resultados de investigación y tranferencia de tecnología del PITTA-Frijol. Publicaciones e impresiones de la Universidad Nacional. Alajuela, Costa Rica. $85 \mathrm{p}$.

CORELlA, J. 1995. Recomendaciones de manejo de la fertilización del cultivo de frijol en Costa Rica. In: Mejoramiento y manejo agronómico del frijol común (Phaseolus vulgaris L.) para adaptación en suelos de bajo fósforo: Memoria del taller internacional sobre bajo fósforo en el cultivo del frijol, 13 al 15 de noviembre de 1995, San José, Costa Rica. pp. 110-138. 
HERNÁNDEZ, J. C.; ACOSTA, M; JIMÉNEZ, S.; PIZARRO, W.; MURILLO, P.; VILLEGAS, O.; MARTINEZ, P.; XATRUCH, C.; VARGAS, L. 2000. Validación del cultivar MD 2324, en dos localidades de Costa Rica. In: Archivos Técnicos. Dirección de Investigaciones Agropecuarias, Ministerio de Agricultura y Ganadería. San José Costa Rica. Sntp.

MORA, B.; FERNÁNDEZ, S.; SOLÓRZANO, A. 1997. Evaluación del Ensayo Centroamericano de Adaptación y Rendimiento (ECAR) en la región Huetar Norte. San José Costa Rica. Ministerio de Agricultura y Ganadería. Dirección de Investigaciones Agropecuarias, Departamento de Fitoprotección. 9 p.

MORALES, A. 1998 . Informe Anual 1997-98, Programa de Frijol. Ministerio de Agricultura y Ganadería, Dirección de Investigaciones Agropecuarias. San José Costa Rica. Snt.

MORALES, A.; HERNÁNDEZ, J. C.; ACOSTA, M; CORDERO, C.; VARGAS, A.; JIMÉNEZ, S.; PIZARRO, W. 1998. Evaluación del Ensayo Nacional de Adaptación y rendimiento (ENAR) de frijol común en tres re- giones de Costa Rica.1997/1998. In: Archivos Técnicos. Dirección de Investigaciones Agropecuarias, Ministerio de Agricultura y Ganadería. San José Costa Rica. Sntp

MORALES, A. 1999. Informe Anual 1998-99, Programa de Frijol. Ministerio de Agricultura y Ganadería, Dirección de Investigaciones Agropecuarias. San José, Costa Rica. Snt.

MORALES, A.; HERNÁNDEZ, J. C.; ACOSTA, M; CORDERO, C.; VARGAS, A.; JIMÉNEZ, S.; PIZARRO, W. 2000. Evaluación del Ensayo Nacional de Adaptación y rendimiento (ENAR) de frijol común en tres regiones de Costa Rica.1998/1999. In: Tercer taller anual de resultados de investigación y tranferencia de tecnología del PITTA-Frijol. Publicaciones e impresiones de la Universidad Nacional. Alajuela, Costa Rica. 85 p.

SALAZAR, J.J. 1999. Situación actual y perspectivas de la producción de frijol en Costa Rica. In: Tercer taller anual de resultados de investigación y transferencia de tecnología del PITTA-Frijol. Alajuela, Costa Rica. p.11-18. 